\section{Coloring Pictures for Electron Microscopists or Elements of Digital Image Manipulation for Students}

V.M.Dusevich, J.H.Purk, J.D.Eick

University of Missouri School of Dentistry, Kansas City, MO DusevichV@umkc.edu

Coloring pictures is an educational exercise, which is fun, and helps develop important skills. Coloring SEM micrographs is especially suitable for electron microscopists. Color micrographs are not just great looking on a lab wall; they inspire both microscopists and students to exercise digital picture manipulation. Many microscopists enjoyed looking at the beautiful color micrographs by D. Scharf, but were frustrated to learn they needed a very particular scanning electron microscope equipped with multiple secondary electron detectors in order to color their own pictures. Fortunately, there are other ways to color SEM micrographs. Most SEMs are equipped with at least two detectors, for secondary and backscattered electrons. Most microscopists use Adobe Photoshop ${ }^{\mathrm{Tw}}$ or similar programs capable of combining several digital pictures into one. Therefore, most microscopists have the means to successfully color pictures. Three somewhat different black and white pictures of the same object could be used as Red, Green, and Blue layers in Photoshop to produce a color picture. "Somewhat different" pictures could be produced by utilizing different detectors, different detector configurations, or different accelerating voltages in the SEM while taking pictures of the same object in the same position at the same magnification. In some cases these pictures would not coincide precisely; this can be corrected by sliding RGB layers slightly relative to each other (in Photoshop), or it can be avoided by utilizing lower magnifications.
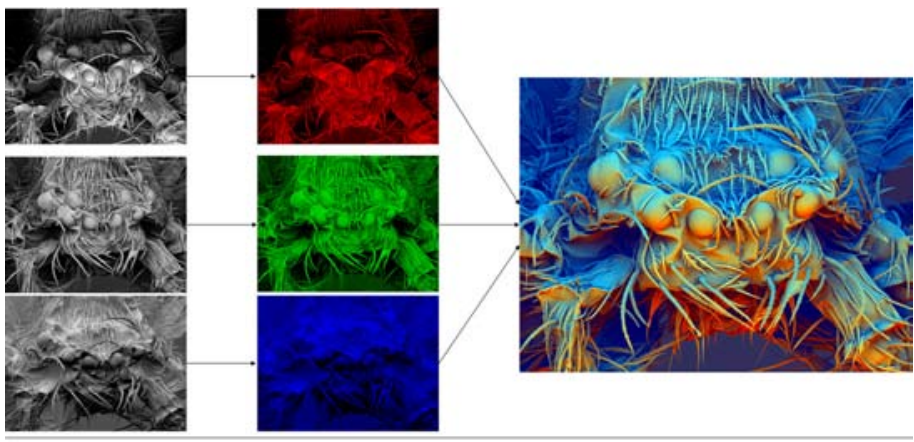

Figure 1. Spider

Figure 1 represents the process of colorizing a portrait of a spider. Just one detector was used, a solid state BSE detector, consisting of two sectors (4 quadrant systems could offer even greater flexibility). Three black and white pictures of the first column of Figure 1 are (from top to bottom): picture taken with sector A of the BSE detector; picture taken with sector B; and picture taken in the so-called "topography" mode, i.e. utilizing the A-B signal.

Utilizing Adobe Photoshop ${ }^{\mathrm{mt}}$, the pictures were converted into Red, Green and Blue layers correspondingly (second column of Figure 1). Each layer was then subjected to various manipulations with Photoshop tools, such as brightness/contrast changes and tweaking with a tool called Levels. Next the picture was "flattened" so that the layers were combined in a single color picture

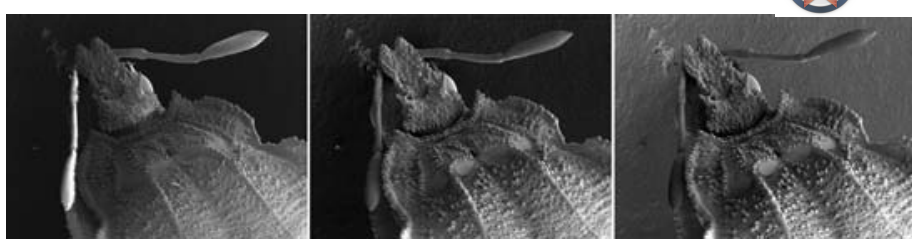

Figure 2. Micrographs of a bug taken at $15 \mathrm{kV}$ (a, left), $5 \mathrm{kV}$ (b, center) and $1 \mathrm{kV}(\mathrm{c}$, right $)$

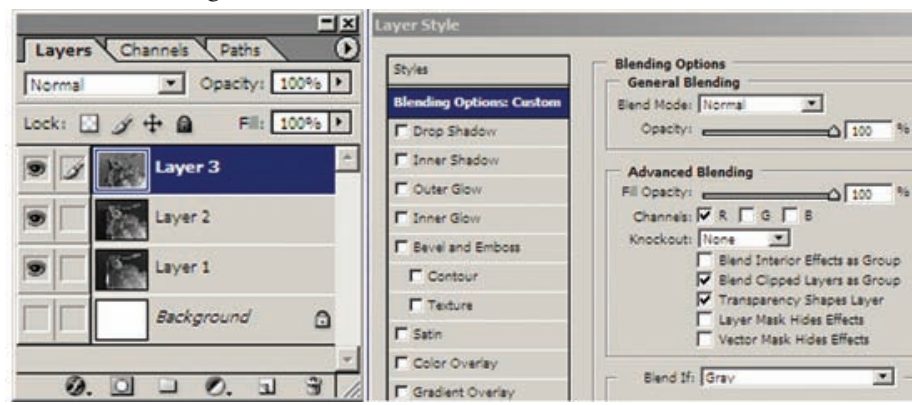

Figure 3. Layers window

Figure 4. Layers Style window

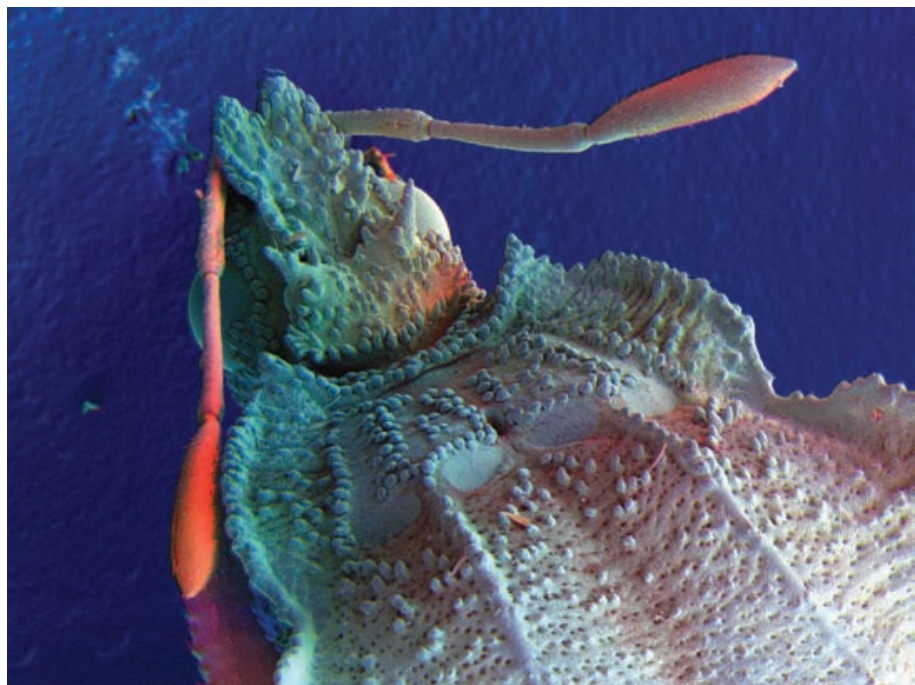

Figure 5. Bug in psuedo color

to decrease the size of the file and make it easier to read in other programs. This picture was again subjected to tweaking with the Hue/saturation, Color balance, Levels, and Curves tools to suit the tastes of the electron microscopist.

The next example is a step by step instruction for coloring the picture of a bug with the help of Adobe Photoshop ${ }^{\text {tw }}$. Three initial black and white pictures were taken with a secondary electron detector at different accelerating voltages: 15,5 and $1 \mathrm{kV}$ (Fig. 2). Some charging could be observed on the left antennae of the bug at $15 \mathrm{kV}$, but it will give a nice accent in the final color picture.

- Open the three initial pictures in Photoshop, select the window with the picture taken at $15 \mathrm{kV}$, in Photoshop menu click on Select, in submenu click $A l l$, and then copy the picture to clipboard (Edit-Copy).

- Open a new window by selecting File - New. The new window will be the same size as the picture on the clipboard.

- Paste the first picture in the new window with Edit - Paste to create Layer 1.

- Highlight the second picture (5 kV), repeat Select - All and Edit - Paste, creating Layer 2.

- Repeat for the third picture $(1 \mathrm{kV})$.

- In the Layers window (Figure 3) click on the Eye icon next to the line Background to make the background invisible. 


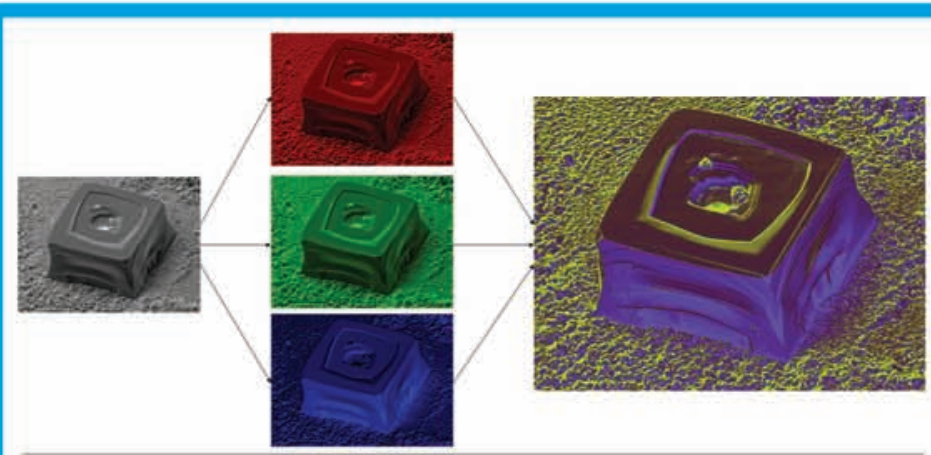

Figure 6. Sodium chloride crystal

- In the Photoshop menu select Image - Mode - RGB Color and in a pop-up window choose Don't Merge; although the image remains visually black and white, it could be colored now.

- Right click on the line Layer 1 and in the submenu choose Blending Options...

- In the pop-up window (Figure 4) in the Channels row, uncheck $\mathrm{G}$ and $\mathrm{B}$ options, making Layer 1 the red channel of the final picture.

- In the same manner make layers 2 and 3 Green and Blue channels.

- Now the picture is colored, but in full resolution (View - Actual Pixels) the blue layer (Layer 3, picture taken at $1 \mathrm{kV}$ ) is misaligned with the other layers. Layer 3 should be selected in the Layers window and then moved with Move Tool (the upper right corner of Photoshop Toolbox) so that it coincides with the Red and Green layers.

- The image can be "flattened" (Layer - Flatten Image).

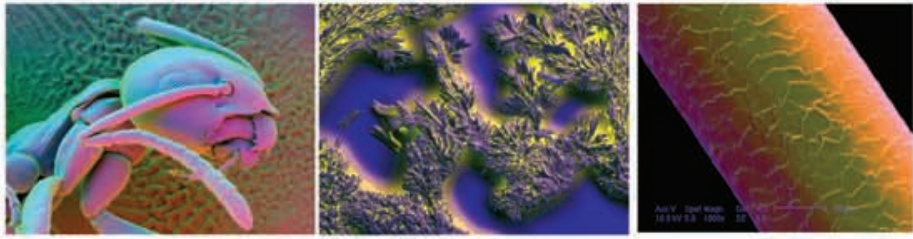

Figure 7, left. Ant

Figure 8. Crystals of sodium bicarbonate

Figure 9, right. Hair

- Some adjustment with the Levels tool (Image - Adjustments Levels) produces the final image (Fig. 5).

Even if the microscopist has no means of producing "somewhat different" pictures of the same object, a color micrograph can still be produced from a single black and white picture, as shown in Figure 6. The same black and white micrograph of a salt crystal was used for all RGB channels (layers), except that in the blue channel an inverted (negative) picture was used. After multiple manipulations with layers and the intermediate picture, the final colored micrograph was produced.

Figure 7, a colored micrograph of an ant, was made from micrographs taken with a secondary electron detector and the sector A and sector B of a BSE detector. The picture from Figure 8, soda crystals, was made with a BSE detector: composition $(A+B)$, topography (A-B), and A modes. For Figure 9 one of the authors donated his hair. The image was composed of micrographs taken with a secondary electron detector, sector A of a BSE detector, and A-B signal of BSE detector.

Supported in part by USPHS Grant K23-DE016324.

\section{When people discover how well the Evactron ${ }^{\circledR}$ De-Comtaminator cleans SEMs they ask: Could You Make it Clean My Kitchen?}

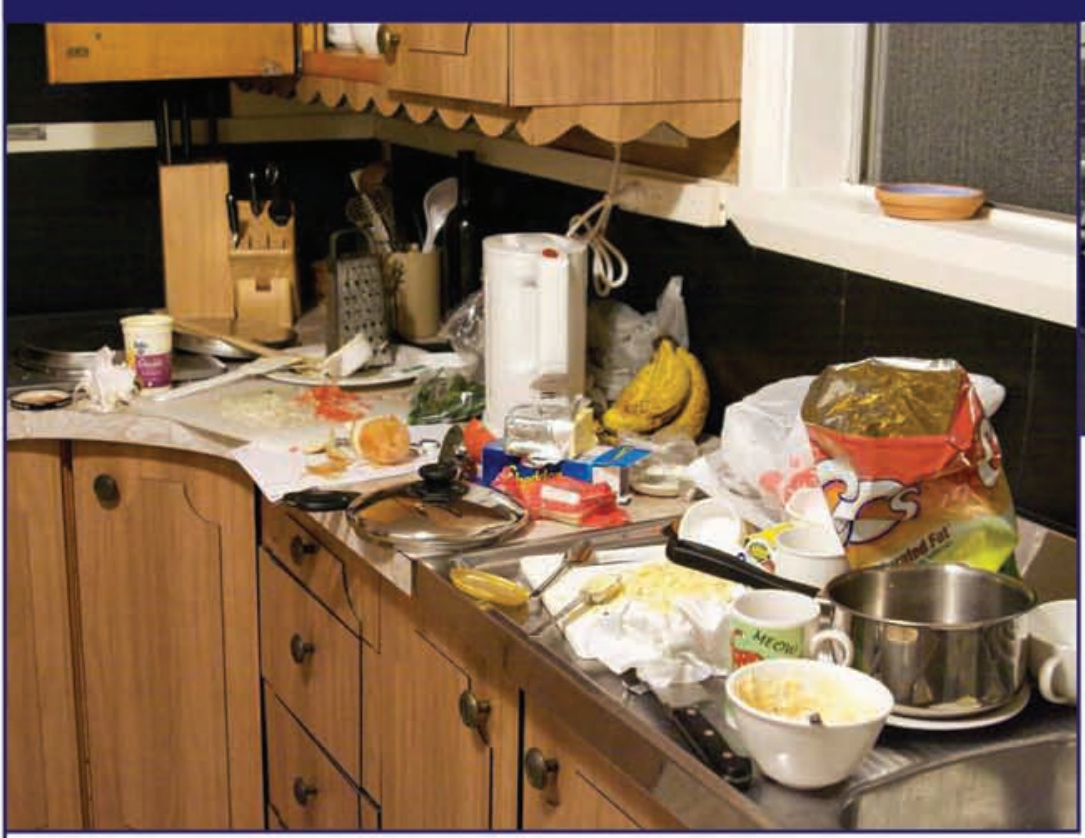

Electron Microscope operators are so impressed with Evactron D-C's gentle and effective cleaning power, they ask us if we can make a bigger Kitchen Evactron with the same powerful oxygen radicals that sweep out grease and oil from SEMs. Fantastic idea. We're considering it!

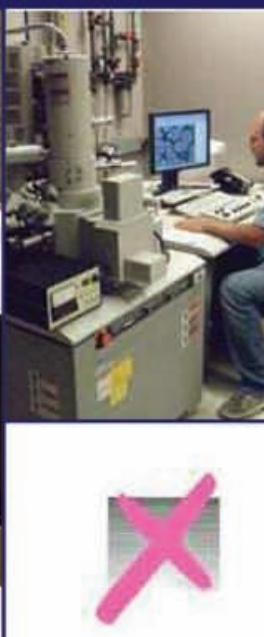

OVER 700

Evactrons D-Cs SOLD WORLDWIDE TO CLEAN SEMs, FIBs, \& HIMs
New Oxygen Radical Source ORS: Now twice the cleaning power!
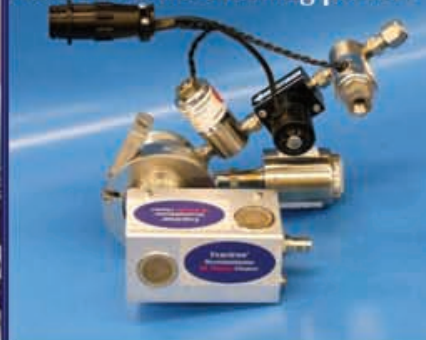

\section{XEI Scientific}

http://evactron.com/

1 (800) 500-0133

General technical inquiries: information@evactron.com

Sales and quotations:

Sales@evactron.com

M\&M Booth 720 